\title{
Charm- and Bottom-Quark Masses: Recent Sum Rule Results
}

\section{J. H. Kühn*}

Institut für Theoretische Teilchenphysik, Karlsruhe Institute for Technology (KIT), Germany

E-mail: johann.kuehn@kit.edu

\begin{abstract}
Recent experimental results on the cross section for electron-positron annihilation into hadrons in the low energy region between roughly $3 \mathrm{GeV}$ and $10 \mathrm{GeV}$, combined with an improved determination of the strong coupling and increasingly precise theory results on sum rules have lead to determinations of charm- and bottom-quark masses with accuracies of around $10 \mathrm{MeV}$. The final results, expressed in the $\overline{\mathrm{MS}}$ scheme, have lead to $m_{c}(3 \mathrm{GeV})=993(8) \mathrm{MeV}$ and $m_{b}\left(m_{b}\right)=4163(16) \mathrm{MeV}$, and are thus among the most precise determinations of these standard model parameters. These results are, furthermore, nicely consistent with completely independent lattice results and allow precise predictions for a number of experimental observables like Higgs decays into charm- or bottom-quarks. A critical analysis of the theoretical and experimental uncertainties of this analysis is presented in the following.
\end{abstract}

Loops and Legs in Quantum Field Theory (LL2018)

29 April 2018 - 04 May 2018

St. Goar, Germany

${ }^{*}$ Speaker. 


\section{Introduction}

The strong coupling constant and the quark masses are the fundamental input parameters of the theory of strong interactions. Quark masses are an essential input for the evaluation of the weak decay rates of heavy mesons and for quarkonium spectroscopy. Decay rates and branching ratios of the Higgs boson depend critically on the masses of the charm and bottom quarks. Last but not least, confronting the predictions for these masses with experiment is an important task for all variants of Grand Unified Theories.

\section{Analytical Results}

Let us recapitulate the main ingredients of the sum rule approach used in the present analysis. Originally the idea has been suggested for the analysis of the charm quark by the ITEP group [1] and, several years later, developed further and applied to the bottom quark [2]. An analysis based on low moments has been performed originally in three- $[3,4]$ and subsequently four-loop $[5,6,7,8,9,10,11,12]$ approximation. Recently new experimental results for the electronic widths of $J / \Psi$ and $\Psi^{\prime}$ in combination with a more precise determination of $\alpha_{s}$ have led to a further shrinking of the uncertainty in the charm quark mass [13].

Let us briefly recall the formalism used to obtain the charm quark mass. The theory prediction of the $n$-th moment of the current-current correlator is obtained from

$$
\mathscr{M}_{n}^{\mathrm{th}}=\left.\frac{12 \pi^{2}}{n !}\left(\frac{\mathrm{d}}{\mathrm{d} q^{2}}\right)^{n} \Pi_{c}\left(q^{2}\right)\right|_{q^{2}=0} .
$$

Here $\Pi_{c}\left(q^{2}\right)$ is the vector correlator with virtual charm-quark loops, which can be cast into the form

$$
\Pi_{c}\left(q^{2}\right)=Q_{c}^{2} \frac{3}{16 \pi^{2}} \sum_{n \geq 0} \bar{C}_{n} z^{n}
$$

with $z=q^{2} /\left(4 m_{c}^{2}\right)$. The $\overline{\mathrm{MS}}$ heavy quark mass at the scale $\mu$ is denoted by $m_{c}=m_{c}(\mu)$ and $Q_{c}=2 / 3$ is the electric charge in units of the elementary charge. The coefficients $\bar{C}_{n}$ depend on $\alpha_{s}$ and on the heavy quark mass through logarithms of the form $l_{m_{Q}}=\ln \left(m_{Q}^{2}(\mu) / \mu^{2}\right)$.

As a perturbative series the coefficients $\bar{C}_{n}$ can be written as

$$
\begin{aligned}
\bar{C}_{n}=\bar{C}_{n}^{(0)} & +\frac{\alpha_{s}(\mu)}{\pi}\left(\bar{C}_{n}^{(10)}+\bar{C}_{n}^{(11)} l_{m_{Q}}\right) \\
& +\left(\frac{\alpha_{s}(\mu)}{\pi}\right)^{2}\left(\bar{C}_{n}^{(20)}+\bar{C}_{n}^{(21)} l_{m_{Q}}+\bar{C}_{n}^{(22)} l_{m_{Q}}^{2}\right) \\
& +\left(\frac{\alpha_{s}(\mu)}{\pi}\right)^{3}\left(\bar{C}_{n}^{(30)}+\bar{C}_{n}^{(31)} l_{m_{Q}}+\bar{C}_{n}^{(32)} l_{m_{Q}}^{2}+\bar{C}_{n}^{(33)} l_{m_{Q}}^{3}\right)+\ldots
\end{aligned}
$$

The terms of order $\alpha_{s}^{2}$ were evaluated up to $n=8$ in Refs. [14, 15, 16] and, subsequently, [17, 18] even up to $n=30$. The four-loop contributions up to $n=3$ were calculated in $[5,6,8,10]$, recently even the moment with $n=4$ was evaluated [12]. Equating theory with the experimentally measured moments

$$
\mathscr{M}_{n}^{\exp }=\int \frac{d s}{s^{n+1}} R_{c}(s)
$$




\begin{tabular}{c|c|cccc|c|c}
\hline$n$ & $m_{c}(3 \mathrm{GeV})$ & $\exp$ & $\alpha_{s}$ & $\mu$ & $\mathrm{np}_{\mathrm{LO}}$ & total & $m_{c}\left(m_{c}\right)$ \\
\hline 1 & 993 & 7 & 4 & 2 & 1 & 8 & 1279 \\
2 & 982 & 4 & 7 & 5 & 1 & 10 & 1269 \\
3 & 982 & 3 & 8 & 6 & 1 & 10 & 1269 \\
4 & 1003 & 2 & 5 & 28 & 1 & 29 & 1288 \\
\hline
\end{tabular}

Table 1: Four-loop results for the charm quark masses, based on the four lowest moments. The errors from experiment, $\alpha_{s}$, the variation of $\mu$ and the nonperturbative piece are listed explicitely.

where $R_{c}=\sigma\left(e^{+} e^{-} \rightarrow c \bar{c}\right) / \sigma\left(e^{+} e^{-} \rightarrow \mu^{+} \mu^{-}\right)$, leads to

$$
m_{c}=\frac{1}{2}\left(\frac{9 Q_{c}^{2}}{4} \frac{\bar{C}_{n}}{\mathscr{M}_{n}^{\exp }}\right)^{\frac{1}{2 n}},
$$

which can be used to extract the charm-quark mass. Even for small values of $n$ the contributions from the low-lying resonances, i.e. the contributions from $J / \Psi$ and $\Psi(2 S)$ are dominant.

\section{Extraction of quark masses}

Most of the experimental input had been compiled and exploited in [7], both for the charm and the bottom case, including, in the case of charm, the tiny contribution from the gluon condensate which leads to a shift of one to two $\mathrm{MeV}$ (For a detailed discussion of this effect see [11]). The most recent analysis for the case of bottom quarks can be found in [9], for charm quarks the most recent update is given in [13]. The most recent results for the charm quark mass can be found in Ref. [13]. For the results based on the lowest four moments one finds the values given in Table 1.

Here $m_{c}(3 \mathrm{GeV})$ denotes the $\overline{\mathrm{MS}}$ mass at the scale of $3 \mathrm{GeV}$, exp the experimental error, $\alpha_{s}$ the strong coupling based on $\alpha_{s}\left(M_{Z}\right)=0.1181 \pm 0.0011$ [19] and $\mathrm{np}_{\mathrm{LO}}$ the error from a variation of the (leading order) nonperturbative terms, based on a gluon condensate of $\left\langle\frac{\alpha_{s}}{\pi} G^{2}\right\rangle=0.006 \pm 0.012$ $\mathrm{GeV}$. For the moment $n=1$, which constitutes our final result, the combined uncertainty amounts to $8 \mathrm{MeV}$. The results for the different $n$-values are nicely mutually consistent and, furthermore, well consistent with earlier determinations based on three-loop analyses. Our final results thus reads

$$
m_{c}(3 \mathrm{GeV})=0.993 \pm 0.008 \mathrm{GeV} \text {, }
$$

which can be evolved to

$$
m_{c}\left(m_{c}\right)=1.279 \pm 0.008 \mathrm{GeV}
$$

A similar analysis has been performed for the case of the bottom quark. The non-perturbative term is practically absent, and the results based on the four lowest moments are given in Table 2. The three lowest moments are of comparable precision. The relative size of the contribution from the continuum above $11.24 \mathrm{GeV}$ which is modelled by perturbative QCD decreases for the higher moments $n=2$ and $n=3$. On the other hand the theory uncertainty, exemplified by the $\mu$ dependence is still acceptable. We therefore adopt the result from $n=2$ (which is roughly between 


\begin{tabular}{c|c|ccc|c|c}
\hline$n$ & $m_{b}(10 \mathrm{GeV})$ & $\exp$ & $\alpha_{s}$ & $\mu$ & total & $m_{b}\left(m_{b}\right)$ \\
\hline 1 & 3597 & 14 & 7 & 2 & 16 & 4151 \\
2 & 3610 & 10 & 12 & 3 & 16 & 4163 \\
3 & 3619 & 8 & 14 & 6 & 18 & 4172 \\
4 & 3631 & 6 & 15 & 20 & 26 & 4183 \\
\hline
\end{tabular}

Table 2: Four-loop results for the bottom quark masses, based on the four lowest moments. The errors from experiment, $\alpha_{s}$ and the variation of $\mu$ are listed explicitely.

the $n=1$ and $n=3$ values and which exhibits the smallest error) as our final result:

$$
\begin{aligned}
m_{b}(10 \mathrm{GeV}) & =3610(16) \mathrm{MeV}, \\
m_{b}\left(m_{b}\right) & =4163(16) \mathrm{MeV}
\end{aligned}
$$

(Note, that here $\alpha_{s}\left(M_{Z}\right)=0.1189 \pm 0.002$ has been adopted). It is straightforward to evolve this result to the renormalization points at $M_{Z}$ and at $m_{t}\left(m_{t}\right)=161.8 \mathrm{GeV}$ :

$$
\begin{aligned}
m_{b}\left(M_{Z}\right) & =2835 \pm 13 \pm 17 \mathrm{MeV}, \\
m_{b}(161.8 \mathrm{GeV}) & =2703 \pm 12 \pm 19 \mathrm{MeV} .
\end{aligned}
$$

To arrive at $m_{t}(161.8 \mathrm{GeV})$, a matching to the $n_{f}=6$ flavour theory has been performed. The first error originates from experiment and from the variation of $\mu$, the second from the variation of $\alpha_{s}$ by \pm 0.002 .

It is of interest to consider the time evolution of this analysis in Fig. (1a) and Fig. (1b), both for charm and bottom masses (red) in comparison with the values in the Particle Data Booklett (blue). The results from 2001 were based on the three loop result Ref. [3], those from 2007 and latter on the four-loop analysis, first with approximate, later with exact results for the higher moments. The convergence of the PDG-result to the values predicted by the present analysis is clearly visible.

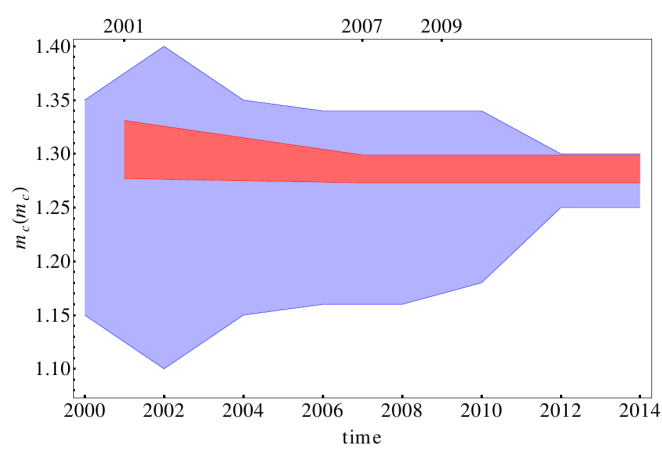

(a) Time evolution of $m_{c}\left(m_{c}\right)$.

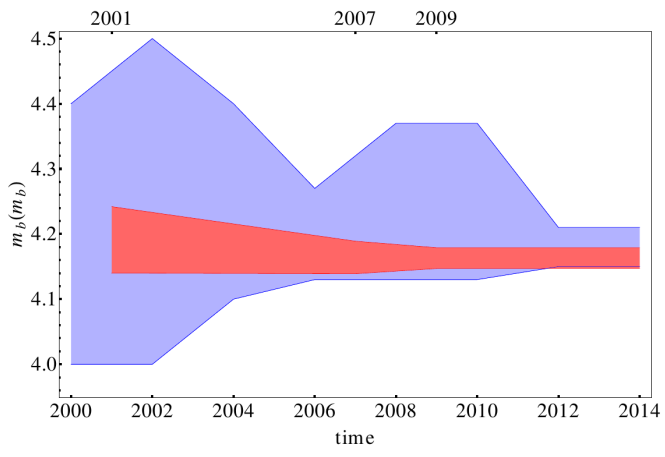

(b) Time evolution of $m_{b}\left(m_{b}\right)$.

The precise value of the strong coupling and the associated errors are often disputed. For this 
reason we also give the explicit dependence of the quark masses on the strong coupling:

$$
\begin{aligned}
m_{c}(3 \mathrm{GeV}) & =\left(993-\frac{\alpha_{s}-0.1181}{0.001} \cdot 5 \pm 16\right) \mathrm{MeV} \\
m_{b}(10 \mathrm{GeV}) & =\left(3610-\frac{\alpha_{s}-0.1189}{0.002} \cdot 12 \pm 11\right) \mathrm{MeV} \\
m_{b}\left(m_{b}\right) & =\left(4163-\frac{\alpha_{s}-0.1189}{0.002} \cdot 12 \pm 11\right) \mathrm{MeV} \\
m_{b}\left(M_{Z}\right) & =\left(2835-\frac{\alpha_{s}-0.1189}{0.002} \cdot 27 \pm 8\right) \mathrm{MeV} \\
m_{b}(161.8 \mathrm{GeV}) & =\left(2703-\frac{\alpha_{s}-0.1189}{0.002} \cdot 28 \pm 8\right) \mathrm{MeV}
\end{aligned}
$$

where $\alpha_{s}=\alpha_{s}\left(M_{Z}\right)$. When considering the ratio of charm- and bottom-quark masses, part of the $\alpha_{s^{-}}$and of the $\mu$-dependence cancels

$$
\frac{m_{c}(3 \mathrm{GeV})}{m_{b}(3 \mathrm{GeV})}=0.2732-\frac{\alpha_{s}-0.1189}{0.002} 0.0014 \pm 0.0028
$$

which might be useful input in ongoing analyses of bottom decays.

\section{Summary}

The most recent analyses of charm- and bottom-quark masses, based on $e^{+} e^{-}$-data combined with sum rules and perturbative QCD in four-loop approximation leads to $m_{c}(3 \mathrm{GeV})=$ $993(8) \mathrm{MeV}$ and $m_{b}(10 \mathrm{GeV})=3610(16) \mathrm{MeV}$. When evolved to the scale of $m_{Q}$, this corresponds to $m_{c}\left(m_{c}\right)=1279(8) \mathrm{MeV}$ and $m_{b}\left(m_{b}\right)=4163(16) \mathrm{MeV}$. These values are nicely consistent with recent results of the HPQCD collaboration, $m_{c}(3 \mathrm{GeV})=985(6) \mathrm{MeV}$ and $m_{b}\left(m_{b}\right)=$ 4162(48) MeV [20] as well as results by the Fermilab Lattice, MILC and TUMQCD collaboration $m_{c}(3 \mathrm{GeV})=984(6) \mathrm{MeV}$ and $m_{b}\left(m_{b}\right)=4197(14) \mathrm{MeV}$ [21].

\section{References}

[1] V. A. Novikov, L. B. Okun, M. A. Shifman, A. I. Vainshtein, M. B. Voloshin and V. I. Zakharov, Phys. Rept. 41 (1978) 1. doi:10.1016/0370-1573(78)90120-5

[2] L. J. Reinders, H. Rubinstein and S. Yazaki, Phys. Rept. 127 (1985) 1. doi:10.1016/0370-1573(85)90065-1

[3] J. H. Kuhn and M. Steinhauser, Nucl. Phys. B 619 (2001) 588 Erratum: [Nucl. Phys. B 640 (2002) 415] doi:10.1016/S0550-3213(01)00499-0, 10.1016/S0550-3213(02)00578-3 [hep-ph/0109084].

[4] J. H. Kuhn and M. Steinhauser, JHEP 0210 (2002) 018 doi:10.1088/1126-6708/2002/10/018 [hep-ph/0209357].

[5] K. G. Chetyrkin, J. H. Kuhn and C. Sturm, Eur. Phys. J. C 48 (2006) 107 doi:10.1140/epjc/s2006-02610-y [hep-ph/0604234].

[6] R. Boughezal, M. Czakon and T. Schutzmeier, Phys. Rev. D 74 (2006) 074006 doi:10.1103/PhysRevD.74.074006 [hep-ph/0605023]. 
[7] J. H. Kuhn, M. Steinhauser and C. Sturm, Nucl. Phys. B 778 (2007) 192 doi:10.1016/j.nuclphysb.2007.04.036 [hep-ph/0702103 [HEP-PH]].

[8] A. Maier, P. Maierhofer and P. Marquard, Phys. Lett. B 669 (2008) 88 doi:10.1016/j.physletb.2008.09.041 [arXiv:0806.3405 [hep-ph]].

[9] K. G. Chetyrkin, J. H. Kuhn, A. Maier, P. Maierhofer, P. Marquard, M. Steinhauser and C. Sturm, Phys. Rev. D 80 (2009) 074010 doi:10.1103/PhysRevD.80.074010 [arXiv:0907.2110 [hep-ph]].

[10] A. Maier, P. Maierhofer, P. Marquard and A. V. Smirnov, Nucl. Phys. B 824 (2010) 1 doi:10.1016/j.nuclphysb.2009.08.011 [arXiv:0907.2117 [hep-ph]].

[11] K. Chetyrkin, J. H. Kuhn, A. Maier, P. Maierhofer, P. Marquard, M. Steinhauser and C. Sturm, Theor. Math. Phys. 170 (2012) 217 doi:10.1007/s11232-012-0024-7 [arXiv:1010.6157 [hep-ph]].

[12] A. Maier and P. Marquard, Phys. Rev. D 97 (2018) no.5, 056016 doi:10.1103/PhysRevD.97.056016 [arXiv:1710.03724 [hep-ph]].

[13] K. G. Chetyrkin, J. H. Kuhn, A. Maier, P. Maierhofer, P. Marquard, M. Steinhauser and C. Sturm, Phys. Rev. D 96 (2017) no.11, 116007 doi:10.1103/PhysRevD.96.116007 [arXiv:1710.04249 [hep-ph]].

[14] K. G. Chetyrkin, J. H. Kuhn and M. Steinhauser, Phys. Lett. B 371 (1996) 93 doi:10.1016/0370-2693(95)01593-0 [hep-ph/9511430].

[15] K. G. Chetyrkin, J. H. Kuhn and M. Steinhauser, Nucl. Phys. B 482 (1996) 213 doi:10.1016/S0550-3213(96)00534-2 [hep-ph/9606230].

[16] K. G. Chetyrkin, J. H. Kuhn and M. Steinhauser, Nucl. Phys. B 505 (1997) 40 doi:10.1016/S0550-3213(97)00481-1 [hep-ph/9705254].

[17] R. Boughezal, M. Czakon and T. Schutzmeier, Nucl. Phys. Proc. Suppl. 160 (2006) 160 doi:10.1016/j.nuclphysbps.2006.09.041 [hep-ph/0607141].

[18] A. Maier, P. Maierhofer and P. Marquard, Nucl. Phys. B 797 (2008) 218 doi:10.1016/j.nuclphysb.2007.12.035 [arXiv:0711.2636 [hep-ph]].

[19] C. Patrignani et al. [Particle Data Group], Chin. Phys. C 40 (2016) no.10, 100001. doi:10.1088/1674-1137/40/10/100001

[20] B. Chakraborty et al., Phys. Rev. D 91 (2015) no.5, 054508 doi:10.1103/PhysRevD.91.054508 [arXiv:1408.4169 [hep-lat]].

[21] A. Bazavov et al. [Fermilab Lattice and MILC and TUMQCD Collaborations], arXiv:1802.04248 [hep-lat]. 